\title{
Fresh Energetic Electron Precipitation at the Noon Sector of the Auroral Magnetosphere during the Recovery Phase of Substorms
}

\author{
L.L. LAZUTIN and V.E. TSIRS
}

Polar Geophysical Institute, Apatity, U.S.S.R.

(Received October 17, 1977)

A statistical study of electron events, using satellite and riometer data, is performed in this work. The satellite used was the polar orbiter 'Cosmos-426' (Vorobiev et al., 1975). A total of 73 passes through the northern auroral zone were investigated. Trapped electrons of energies $>20,>60,>250$ and $\sim 63 \mathrm{keV}$, and precipitating electrons of energy, $\sim 62 \mathrm{keV}$ were measured in the local time sector of 10 to 19 MLT. Apatity $\left(\phi=63.3^{\circ}\right)$ and Loparskaya $\left(\phi=64^{\circ}\right)$ riometer data were used for the period 1969-1972. $A L$ indices and magnetograms were used to indicate substorm phase.

Figure 1 shows the regions of enhanced isotropic electron fluxes with energy $\sim 63 \mathrm{keV}$ for three disturbed periods: August 2, November 12 and August 31 of 1971 (arrows indicate the $E>250 \mathrm{keV}$ electron trapping boundary). One can see that intense isotropic fluxes localized near midday during the moderate disturbances

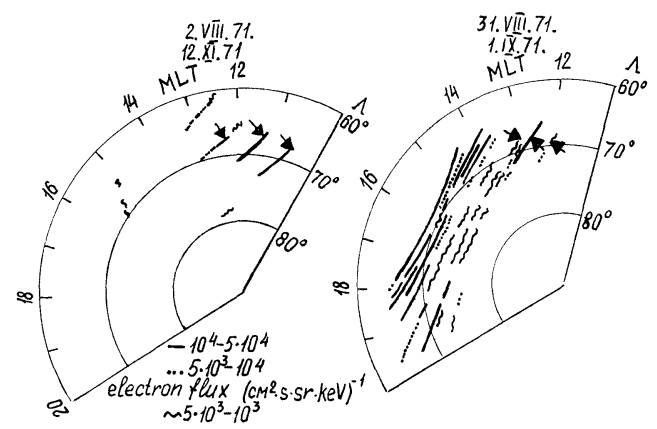

Fig. 1. The regions of enhanced isotropic electron fluxes with energy $\sim 63 \mathrm{keV}$.

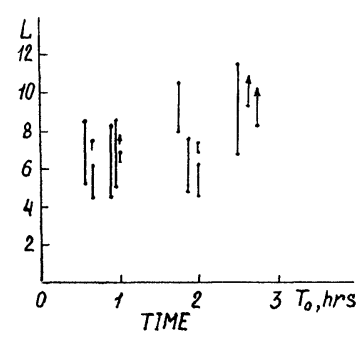

Fig. 2. The latitudinal extension $(\Delta L)$ of isotropic fluxes versus the interval between onset of flux and onset of substorm $\left(T_{0}\right)$. 
of August 2 and November 11-12 and spread over a wide region toward the evening sector during the intense substorm of August 31. Structured isotropic flux increases were measured only during the recovery phase. Figure 2 shows the latitudinal extension of increases $(\Delta L)$ versus the time interval between the measurement and the substorm onset in the night sector. (The trend of a poleward shift of the precipitation region with time during substorm recovery is evident.) Analysis of spectral data shows that, in the region of fresh particle precipitation, the spectrum softens relative to the stably trapped electron population. The $E$-folding energy has a value of $12-15$ and $>20 \mathrm{keV}$ respectively. All fine structure variations are similar in the $E=62$ and $E>20 \mathrm{keV}$ channels.

It is well known that, during daytime, riometers sometime register sharply structured absorption events, similar to active nighttime $F$-type ones. Analysis of individual cases and a statistical approach clearly show an increase of auroral absorption simultaneous with the beginning of the recovery phase of substorm both for isolated and long-lasting events.

Isotropic electron increases were observed in the dayside sector of the auroral magnetosphere with a soft energy spectrum and no energy dispersion within the interval $20-60 \mathrm{keV}$, similar to locally accelerated midnight events. These events were observed to be randomly distributed within the time interval from $30 \mathrm{~min}$ to $3 \mathrm{hr}$ after the beginning of the active phase of substorm, and closely connected to the beginning of the recovery phase of substorms. These features are taken as evidence of the localized fresh-acceleration of the electrons. We believe that the acceleration process may be caused by an electric field induced by the rapidly changing magnetic field as the lobe of the magnetosphere recovers from its previously eroded configuration.

\section{REFERENCE}

Vorobiev, V.A., S.N. KuZnetzov, I.N. Lisenko, B.I. SAVIN, and V.G. StolpovskiJ, Earth's Satellite 'Kosmos-426,' Kosmich. Issled., 5, 778-783, 1975. 